\title{
Mochii ISS-NL: Electron Microscopy Has Arrived at the International Space Station
}

Christopher Own ${ }^{1}$, James Martinez ${ }^{2}$, Theodore DeRego ${ }^{1}$, Lawrence Own ${ }^{1}$, Zachary Morales ${ }^{1}$, Katherine Thomas-Keprta ${ }^{3}$, Zia Rahman ${ }^{3}$ and Donald Pettit ${ }^{3}$

${ }^{1}$ Voxa, Seattle, Washington, United States, ${ }^{2}$ NASA Johnson Space Center / Structural Engineering Division, Houston, Texas, United States, ${ }^{3}$ NASA Johnson Space Center, Houston, Texas, United States

An electron microscope is now, for the first time, in Earth orbit aboard the International Space Station (ISS). A Mochii ${ }^{\mathrm{TM}}$ portable electron microscope was delivered to ISS via the Cygnus spacecraft on NASA's NG-13 commercial resupply mission (Fig. 1), and is now traveling at 17,136 mph, about $250 \mathrm{mi}$ above the Earth's surface [1]. As a new addition to the ISS National Laboratory, Mochii ISS-NL will support novel in-situ microgravity science and engineering in low Earth orbit (LEO) for the benefit of humankind [2].

The ISS is currently the only manned facility available that supports research in the unique continuous microgravity environment of LEO. Equipment and materials on board ISS are available for use by the public - researchers, commercial companies, and educational institutions - to perform experiments and engineering in microgravity. Major motivations for providing sophisticated capabilities like electron microscopy on ISS include:

- Measurement of sample response to unique environments (radiation, microgravity, ionization)

- Progress of phenomena over time (crystal evolution, cell growth)

- Needs from crew or vehicle (telemetry, safety assessment)

- Samples that may be altered or destroyed during return to Earth (precipitation experiments, fragile or time-sensitive samples)

The addition of the Mochii ISS-NL facility to the ISS toolset enables a broad range of scientific inquiries that are relatively routine on Earth but have until now been impossible to perform on-vehicle in the space environment. The high native resolution and chemical ID offered by scanning electron microscopy (SEM) equipped with energy-dispersive X-ray spectroscopy (EDS) serves broad areas of science and engineering. Mochii ISS-NL is a joint project between NASA's Structural Engineering, Astromaterials divisions, the Astronaut office, the ISS program, and commercial company Voxa. It provides for a modified commercial off-the-shelf (COTS) collaborative multi-user tablet-controlled instrument integrating sub-micrometer imaging and EDS into a coffee-maker sized form factor, to be installed in the Japanese Experiment Module (JEM) [3]. (Fig. 2)

After initial demonstration, on-orbit investigations expected to be conducted with Mochii include materials studies such as microgravity crystal growth, in-space manufacturing, and fracture analyses. Mochii is also planned to perform morphological, textural and chemical characterization of extraterrestrial samples and impact craters from space environment exposure. Longer-term, we expect to also process biological samples from living creatures. Concurrently, Mochii will enhance crew and vehicle safety by rapidly and accurately identifying microscopic mission threats, especially in time-critical situations where debris from damaged systems cannot be sent back. Examples of such critical situations are crewmember Luca Parmitano's waterlogged extra-vehicular activity (EVA) suit in 2013 and the ISS starboard solar 
alpha rotary joint failure in 2007 where chemical analysis of microscopic particles played a central role in identifying point of failure, source, and problem resolution [4-5].

To reach this point, Mochii has undergone rigorous flight certifications, including testing and verifications in two categories: vehicle integration and safety, and functional/science. Integration and safety requirements ensure that payloads are safe for crew and vehicle, and are required for transport of payloads and for orbital operations once on-board. These tests included power quality, EMI/EMC, radiation safety, acoustic, thermal, command \& data handling, and human factors integration. The second class of verifications (functional/science) included reference sample analyses, analog testing of on-orbit prototype procedures in the NEEMO XXIII underwater mission, magnetic testing, and flight vibration survival testing [6]. Due to sensitivity of electron optical instrumentation, iterative vibration testing was conducted over several design cycles, with final acceptance testing conducted in flight foam to achieve two-vehicle launch certification (Cygnus and Dragon).

As the first SEM moving at high speeds around the Earth, Mochii required special considerations to counter potential beam drift from continuously changing magnetic fields. The Earth's field in LEO varies by $200-650 \mathrm{mG}$ over the $\sim 90 \mathrm{~min}$ ISS orbit (with gradients up to $25 \mathrm{mG} / \mathrm{min}$ ) and requires significant measures to avoid beam shifts and distortions [7]. Coauthors at Voxa constructed a custom magnetic test apparatus called Monarch ${ }^{\mathrm{TM}}$ to perform iterative testing of magnetic shielding in Mochii in order to achieve beam drift levels expected to support sub-micrometer EDS microanalysis in LEO.

Engineering, geological, and calibration samples were prepared in a compact sample preparation field kit. Reference analyses on these were performed using both Mochii and larger laboratory SEM's for comparison with upcoming orbital results. We now look forward to conducting the first demonstration of microanalysis on ISS, which we expect will open new opportunities to explore phenomena in microgravity at the micro- and nanoscales.

Beyond routine microgravity research, Mochii ISS-NL is intended to serve as a research platform for future space exploration. With its current certification, Mochii lends itself toward use on missions on crewed vehicles extending outside of LEO, from upcoming moon missions on the Gateway vehicle to interplanetary expeditions. The technology platform will also enable early testing for utilization on other worlds. We will be exploring use of microanalysis capability on both crewed and unmanned robotic missions on the surface, with applications ranging from sample return to in-situ resource utilization (ISRU) where future crews will be looking to use natural resources to produce tools, vehicles, and fuel before return to Earth. [8] 


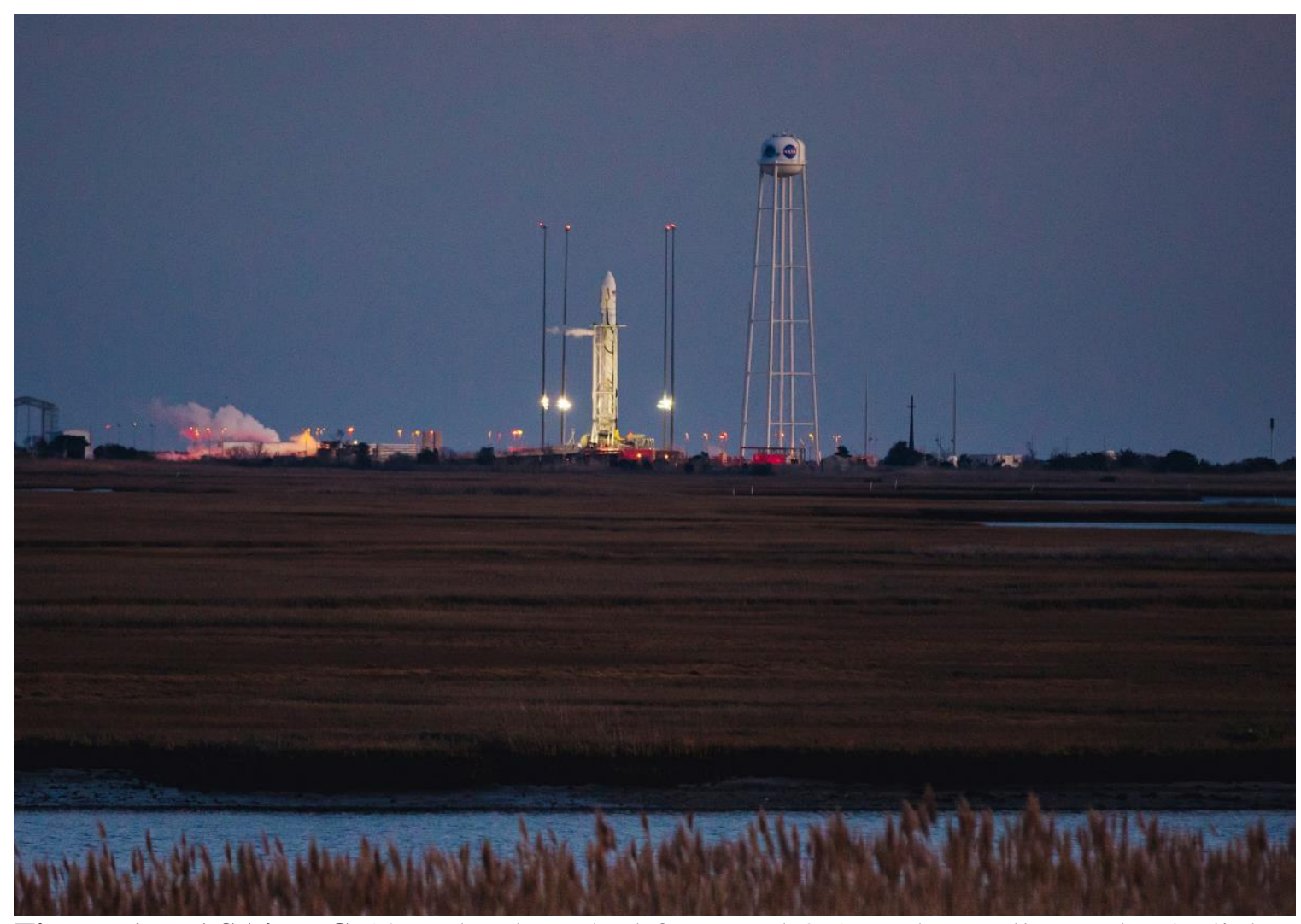

Figure 1. NASA's NG-13 rocket launched from Pad 0-A at the Wallops Island Flight Facility in VA, carrying the Mochii ISS-NL spectroscopic scanning electron microscope investigation, plus 7500 pounds of other experiments, supplies, and food to the ISS on Feb 15, 2020.

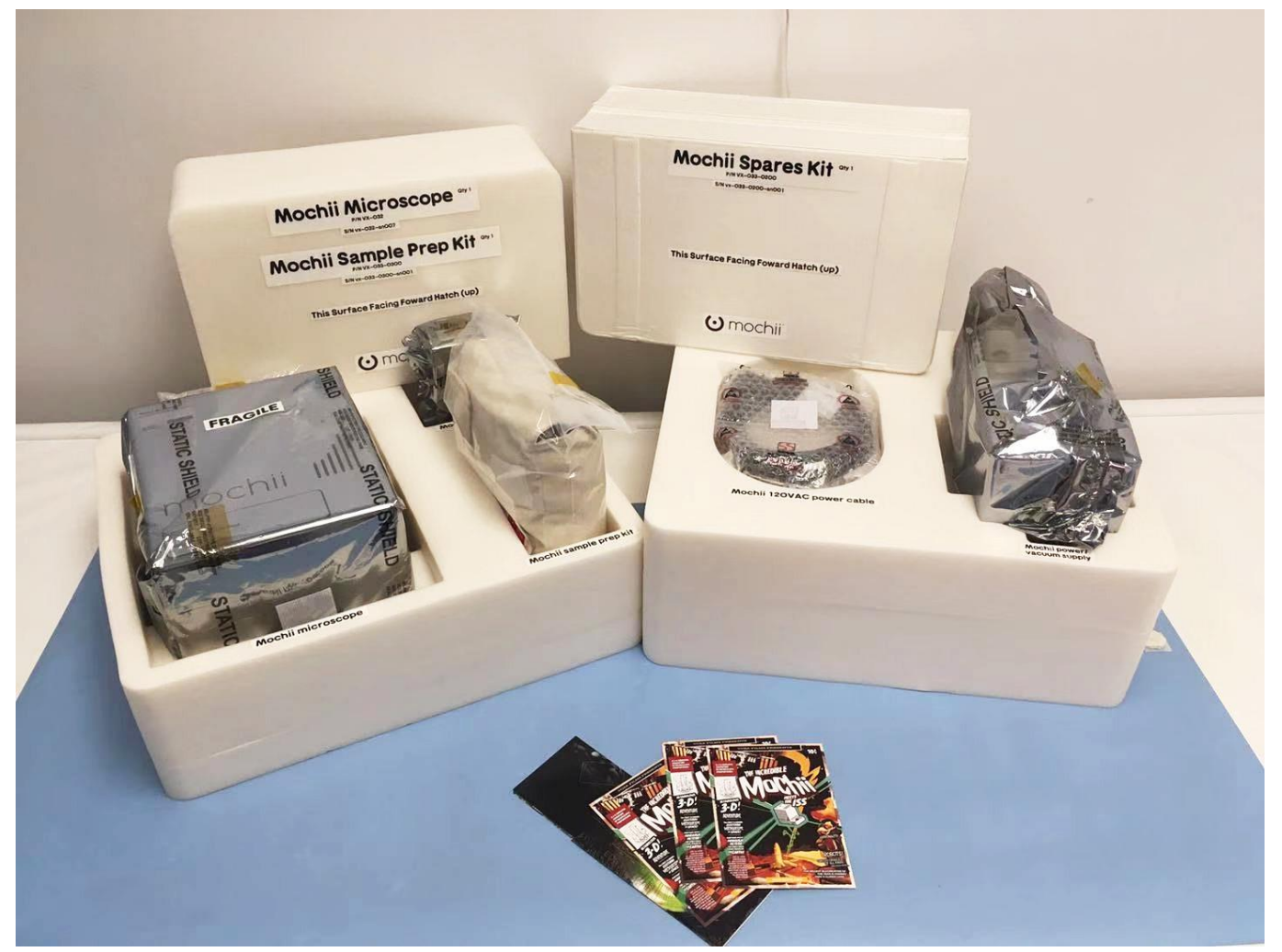


Figure 2. Mochii microscope sealed and packed in foam at handover in Dec 2019, at the ISS Cargo Mission Contract processing center in Houston, TX.

\section{References}

[1] https://www.cbsnews.com/news/antares-rocket-boosts-space-station-cargo-ship-into-orbit-2020-02$15 /$

[2] https://www.nasa.gov/mission_pages/station/research/experiments/explorer/Facility.html?\#id=7657

[3] Own, et al, LPSC 2019 (https://www.hou.usra.edu/meetings/lpsc2019/pdf/3238.pdf).

[4] https://ntrs.nasa.gov/archive/nasa/casi.ntrs.nasa.gov/20110015384.pdf

[5]

https://www.nasa.gov/sites/default/files/files/Suit_Water_Intrusion_Mishap_Investigation_Report.pdf

[6] Own, et al, M\&M 2019 (https://www.microscopy.org/MandM/2019/program/abstracts/PDP-6.pdf)

[7] Martinez, et al. Microscopy \& Microanalysis 25 S2 (2019), 700-701.

[8] This work was supported by Voxa, NASA, and Jacobs. 